\title{
Incorporation of Naked Peptide Nucleic Acids into Liposomes Leads to Fast and Efficient Delivery
}

\author{
Concetta Avitabile, ${ }^{\dagger, \#}$ Antonella Accardo, ${ }^{\ddagger},, \#$ Paola Ringhieri, ${ }^{\ddagger}$ Giancarlo Morelli, ${ }^{\ddagger}, \S$ Michele Saviano, ${ }^{\S, \|}$
}

Giulia Montagner, ${ }^{\perp}$ Enrica Fabbri, ${ }^{\perp}$ Eleonora Gallerani, ${ }^{\perp}$ Roberto Gambari, ${ }^{\perp}$ and Alessandra Romanelli* ${ }^{\ddagger}, \S$

${ }^{\dagger}$ Institute of Biostructure and Bioimaging (IBB), CNR, via Mezzocannone 16, 80134 Napoli, Italy

${ }^{\ddagger}$ Department of Pharmacy, University of Naples "Federico II”, via Mezzocannone 16, 80134 Napoli, Italy

${ }^{\S}$ Interuniversity Research Centre on Bioactive Peptides (CIRPeB), via Mezzocannone 16, 80134 Napoli, Italy

"Institute of Crystallography (IC), CNR, Via Amendola 122, 70126 Bari, Italy

${ }^{\perp}$ Department of Life Sciences and Biotechnologies, University of Ferrara, via Fossato di Mortara 74, 44121 Ferrara, Italy

\section{Supporting Information}

ABSTRACT: The delivery of peptide nucleic acids (PNAs) to cells is a very challenging task. We report here that a liposomal formulation composed of egg $\mathrm{PC} /$ cholesterol/DSPEPEG2000 can be loaded, according to different encapsulation techniques, with PNA or fluorescent PNA oligomers. PNA loaded liposomes efficiently and quickly promote the uptake of a PNA targeting the microRNA miR-210 in human erythroleukemic K562 cells. By using this innovative delivery system for PNA, down-regulation of miR-210 is achieved at a low PNA concentration.

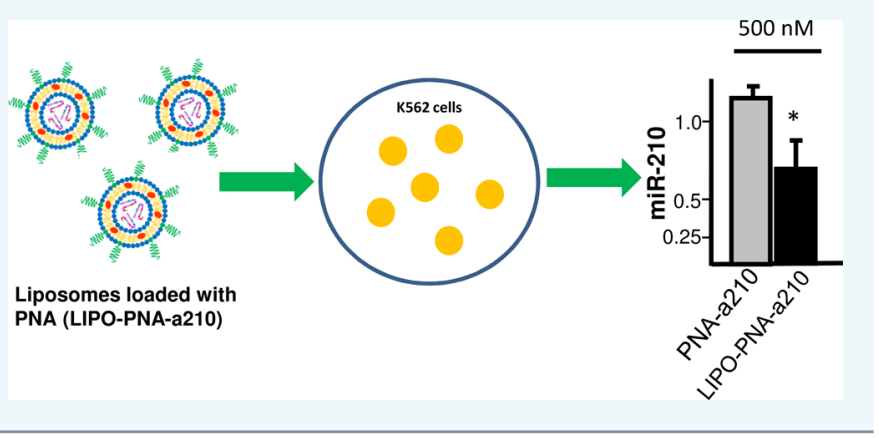

branes; ${ }^{12,13}$ negatively charged moieties such as sulfate and phosphonate installed on the PNA backbone allowed delivery of PNA by standard cationic liposomes. ${ }^{14,15}$

An alternative strategy to improve the cellular uptake of PNAs is based on their conjugation to carrier peptides. Positively charged peptides such as Tat, NLS, and polyarginine are usually conjugated at one end of the PNA; the resulting molecules show an increased ability to be internalized by target cells. ${ }^{16}$ In a recent application, the delivery of a PNA to a tumor has been achieved by a peptide specific for acidic microenvironments, which are typical of tumors: the pHLIP peptide, which forms a transmembrane $\alpha$ helix at low $\mathrm{pH}$, has been conjugated to an antimiR-155 PNA and has been demonstrated to inhibit miR-155 in a mouse lymphoma model. ${ }^{17}$ Silica nanoparticles have been linked to PNA by disulfide bond to achieve delivery and release of antisense PNAs in HeLa cells; ${ }^{18}$ biodegradable PLGA formulations have been successfully employed for the delivery in vivo of PNA-polylysine conjugates in hematopoietic cells. ${ }^{19}$ The main drawback of the cited strategies derives from the need of synthesizing special building blocks to be used in the solid phase synthesis or to conjugate the carriers on solid phase or in solution, ending up in very large molecules.

Received: March 25, 2015

Revised: July 7, 2015

Published: July 15, 2015 


\section{Empty liposomes formulation}

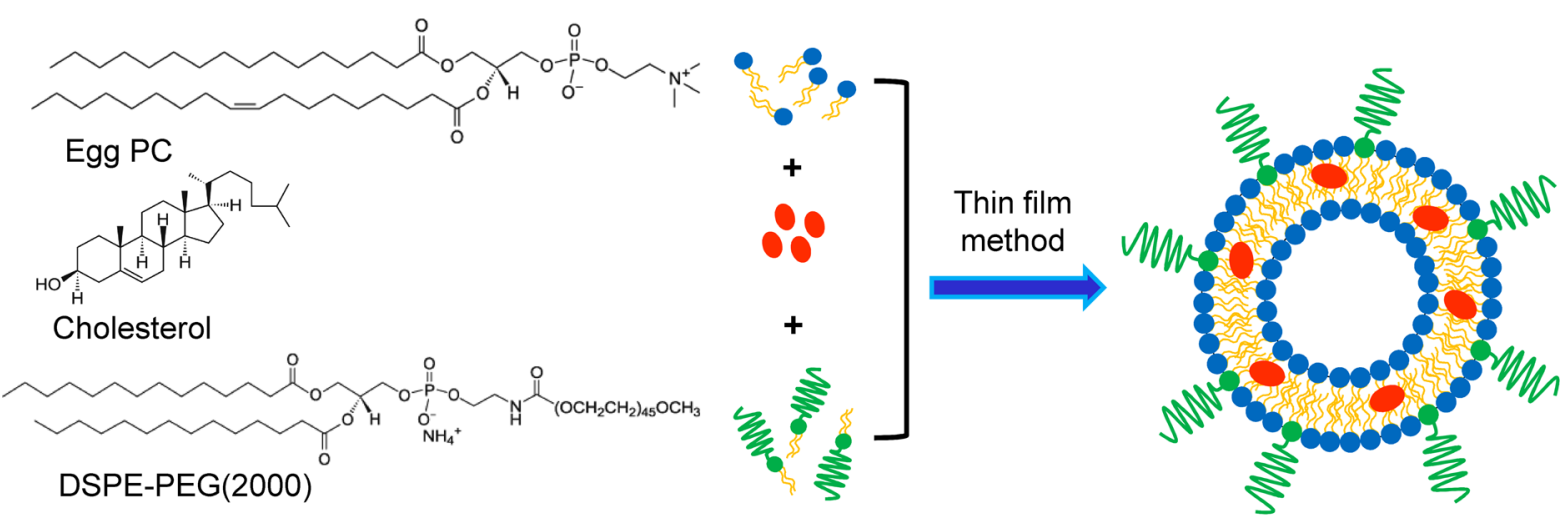

\section{PNA encapsulation}

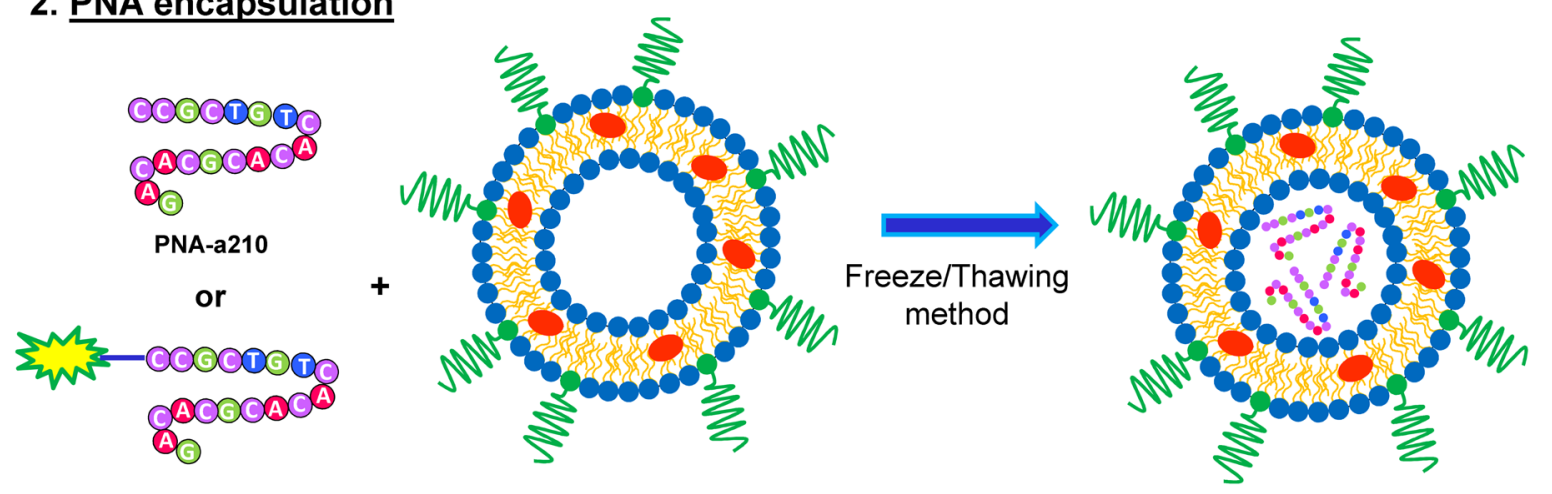

FLUO-PNA-a210

Figure 1. Preparation of PNA loaded liposomes according to the freeze/thawing encapsulation method. In the first step the mixture of lipids was dried under vacuum to form a lipid film, then the PNA solution is added to the vial and liposomes encapsulating PNA are obtained.

Usually nucleic acids are delivered into cells by cationic liposomes, such as $N$-[1-dioleyloxy)propyl]- $N, N, N$-trimethylammonium (DOTMA) and dioleoylphophatidylethanolamine (DOPE) in a 1:1 phospholipid mixture, and 2,3-dioleyloxy- $N$ [2(sperminecarboxamido)ethyl]-N,N-dimethyl-1-propanammonium trifluoroacetate (DOTAP). The cationic head of these liposomes allows the complexation of the negatively charged phosphate groups of DNA/RNA, the linker region affects the stability and biodegradability of the liposome while the hydrophobic region anchors the cationic lipid into the bilayer. ${ }^{20}$ Unfortunately, cationic liposomes are not suitable for the delivery of PNAs, unless they are hybridized or conjugated to DNA. $^{21-23}$

In this paper we show that peptide nucleic acid oligomers can be incorporated into egg phosphatidylcholine/cholesterol/1,2distearoyl-sn-glycero-3-phosphoethanolamine- $N$ - [carbonylmethoxy(polyethylene glycol)2000] (egg PC/Chol/DSPEPEG2000) liposomes and efficiently delivered to cells.

This finding has important implications for in vivo use of these oligonucleotide analogues: PNA oligomers made exclusively of commercially available PNA monomers are delivered to cells, without further modifications, and can exert their function at low concentrations.

\section{RESULTS}

Liposome Preparation and Structural Characterization. PNA-antimiR210 and FLUO-PNA-antimiR210 derivatives were encapsulated in mixed egg PC/Chol/DSPEPEG2000 liposomes at 47/47/6 molar ratio. Loading of the
PNAs molecules was assessed by exploring three different methods: thin film method/sonication (a), freeze/thawing (b), and SNALPs (c). Empty liposomes were also prepared according to these procedures.

Figure 1 reports a schematic representation of the freeze/ thawing encapsulation method.

The liposomes containing PNA-a210 and its fluorescent derivative, namely, LIPO-PNA-a210 and LIPO-FLUO-PNAa210, were characterized by DLS measurements from the structural point of view and by UV-vis spectroscopy for definition of encapsulation properties. Structural data and encapsulation efficiency are reported in Table 1; DLS profiles of PNA loaded liposomes are reported in Figure 2.

Table 1. Structural Parameters (Hydrodynamic Diameters, Polydispersity Indexes, PDI, and Diffusion Coefficients, D) from Dynamic Light Scattering Measurements and Encapsulation Efficiency (\%) of Liposomes Prepared by Methods a, b, and c

\begin{tabular}{|c|c|c|c|c|c|}
\hline method & compound & $\begin{array}{c}\text { diameter } \\
(\mathrm{nm}) \pm \\
\text { S.D. }\end{array}$ & PDI & $\underset{\times 10^{-12}}{D}\left[\mathrm{~m}^{2} \mathrm{~s}^{-1}\right]$ & $\begin{array}{c}\mathrm{EE} \\
(\%)\end{array}$ \\
\hline \multirow[t]{2}{*}{ (a) } & PNA-a210 & $85 \pm 46$ & 0.148 & $5.8 \pm 3.1$ & 1.69 \\
\hline & FLUO-PNA-a-210 & $78 \pm 32$ & 0.132 & $6.2 \pm 2.5$ & 0.67 \\
\hline \multirow[t]{2}{*}{ (b) } & PNA-a210 & $100 \pm 35$ & 0.087 & $4.9 \pm 1.7$ & 1.75 \\
\hline & FLUO-PNA-a-210 & $95 \pm 28$ & 0.054 & $5.1 \pm 1.5$ & 0.73 \\
\hline \multirow[t]{2}{*}{ (c) } & PNA-a210 & $114 \pm 58$ & 0.160 & $4.3 \pm 2.2$ & 1.85 \\
\hline & FLUO-PNA-a-210 & $102 \pm 49$ & 0.148 & $4.8 \pm 2.3$ & 0.82 \\
\hline
\end{tabular}




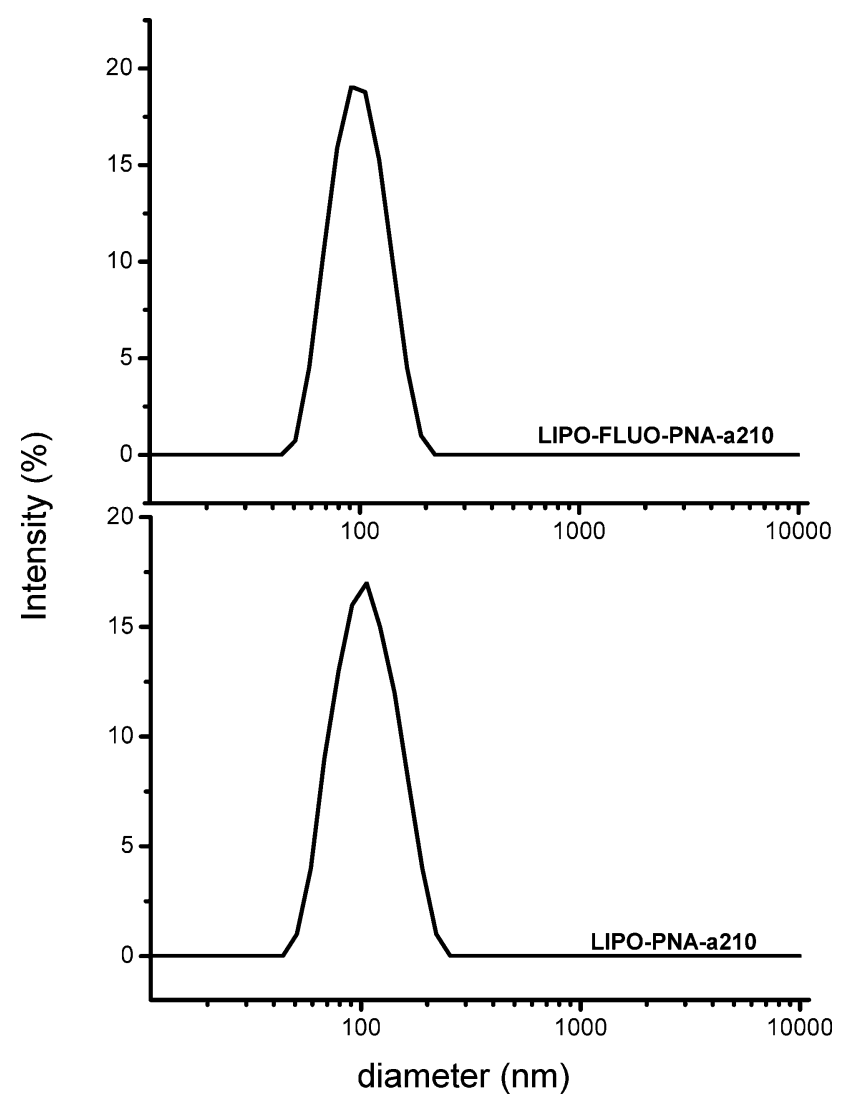

Figure 2. Dynamic light-scattering plots of liposomes encapsulating PNA-antimiR210 (LIPO PNA-a210) and FLUO-PNA-antimiR210 (LIPO FLUO-PNA-a210) prepared according to the freeze/thawing method.

From the inspection of Table 1, only slight differences can be observed between the mean diameter values obtained with the three loading procedures $(78-114 \mathrm{~nm})$. In all cases, samples encapsulating FLUO-PNA-antimiR210 showed a lower size ( $\sim 10 \%$ of decrease) with respect to those encapsulating PNAantimiR210. On the other hand, a significant decrease of the polydispersity index is worth noting for both preparations obtained with the loading procedure (b). Moreover, any substantial difference has been revealed in the encapsulation efficiency achieved with the three procedures (see Table 1). An increase in the encapsulation efficiency can be obtained by the postinsertion method (Table S1).

As expected, empty liposomes, containing $47 \mathrm{~mol} \%$ of PC zwitterionic phospholipid and $6 \mathrm{~mol} \%$ of DSPE-PEG2000 anionic phospholipid, have a $Z$ potential value of $-31.13 \mathrm{mV}$. After loading with PNA-a210 or with its fluorescent derivative FLUO-PNA-a210, $\mathrm{Z}$ values were -26.4 and $-23.6 \mathrm{mV}$, respectively. PNA loaded liposomes can be lyophilized and stored at $-20{ }^{\circ} \mathrm{C}$; neither leakage of PNAs nor changes in the liposome dimensions and PDI are observed upon resuspension in water and sonication of lyophilized liposomes (Table S2).

Stability of liposomes loaded with PNAs was also assessed in fetal bovine serum. After incubation with serum, liposomal suspension dimensions were monitored by DLS measurements for $72 \mathrm{~h}$. As indicated in Figure 3, any variation in the liposomal diameter occurs, thus indicating its high stability under these experimental conditions.

Liposomes labeled with rhodamine encapsulating the FLUOPNA-antimiR210 (Rho-LIPO-FLUO-PNA-a210 were also

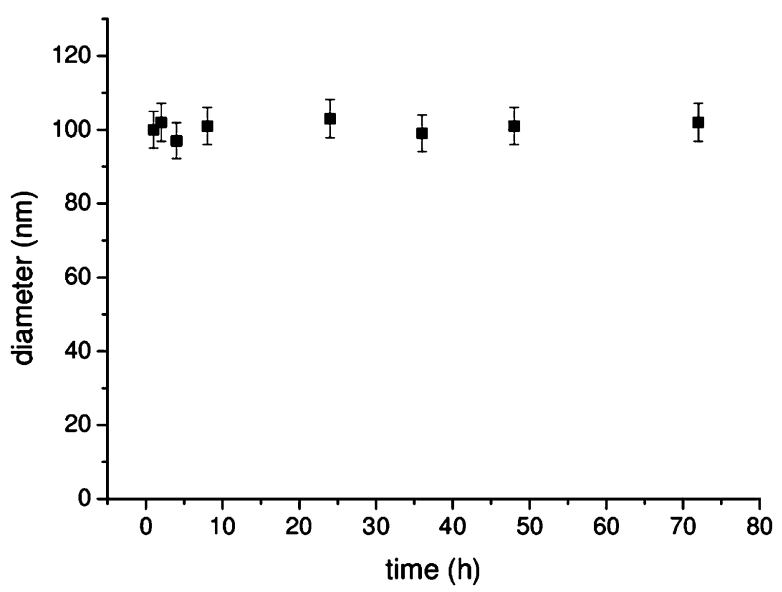

Figure 3. Stability of LIPO-PNA-a210 in $90 \%$ fetal bovine serum.

prepared and employed to detect the cellular uptake of PNAa210 in K562 cells by confocal microscopy.

Uptake of PNAs by K562 Cells. Uptake studies were carried out employing K562 cells as experimental model system, using the following fluorescently labeled molecules: FLUO-PNA-a210, FLUO-PNA-a210-R8 (a PNA targeting miR-210 and conjugated to a polyarginine $\mathrm{R} 8$ sequence), and the LIPO-FLUO-PNA-a210 formulation. Figure 4 (panels A and B) shows a first set of experiments in which K562 cells were cultured for $4 \mathrm{~h}$ in the presence of $2 \mu \mathrm{M}$ and $4 \mu \mathrm{M}$ FLUO-PNA-a210, FLUO-PNA-a210-R8, and LIPO-FLUOPNA-a210 and were analyzed by FACS.

As expected, low uptake levels were found when FLUOPNA-a210 was employed. This is perfectly in agreement with results previously published by our research group demonstrating that the PNA-a210 is internalized by target cells only if it is linked to an octarginine R8 peptide. ${ }^{24,25}$ The summary of the time course of the uptake is shown in Figure 4C, which indicates that uptake of LIPO-PNA-a210 is fast and a plateau level is reached between 4 and $24 \mathrm{~h}$ culture period. No appreciable uptake of FLUO-PNA-a210 by K562 cells was detectable even after $48 \mathrm{~h}$ of culture. The representative microscopic analyses shown in Figure 4 (panels D-F) demonstrate that no evidence of intracellular uptake of FLUO-PNA-a210 is detectable (Figure 4E). By contrast, liposomes carrying the FLUO-PNA-a210 bind to the cellular membrane and, when internalized, are located mostly in the intracellular matrix (Figure 4D and F). When staining of nuclei is performed (Figure 4F,b), we can conclude that no liposomemediated delivery of FLUO-PNA-a210 is detectable at the nuclear level, and the localization is mostly cytoplasmic. To conclusively demonstrate intracellular localization of LIPOPNA-a210, confocal microscopic analysis was performed on living cells (Figure 5). Figure 5A shows that, in agreement with the FACS data shown on Figure 4A and the microscopic analysis shown in Figure 4E, no uptake of FLUO-PNA-a210 is detectable, since almost all of the fluorescence was found outside the cells. On the contrary, when rhodamine-labeled liposomes carrying FLUO-PNA-a210 (Rho-LIPO-FLUOPNA) were employed, most of the fluorescence was found within the cells, formally demonstrating liposome-mediated uptake of the PNA-a210 (Figure 5B). In any case, these results were considered a promising background to investigate possible effects on miR-210 in LIPO-FLUO-PNA-a210 treated cells. Taken together, the data shown in Figures 4 and 5 strongly 

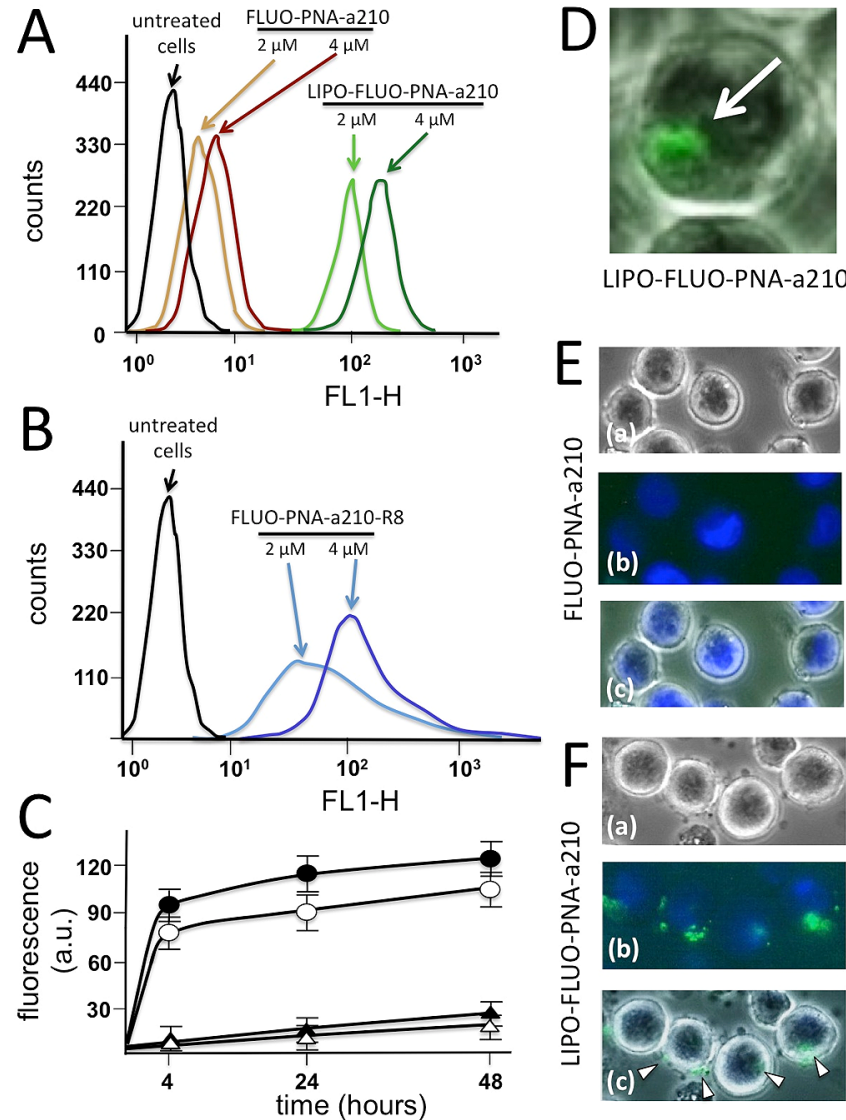

LIPO-FLUO-PNA-a210
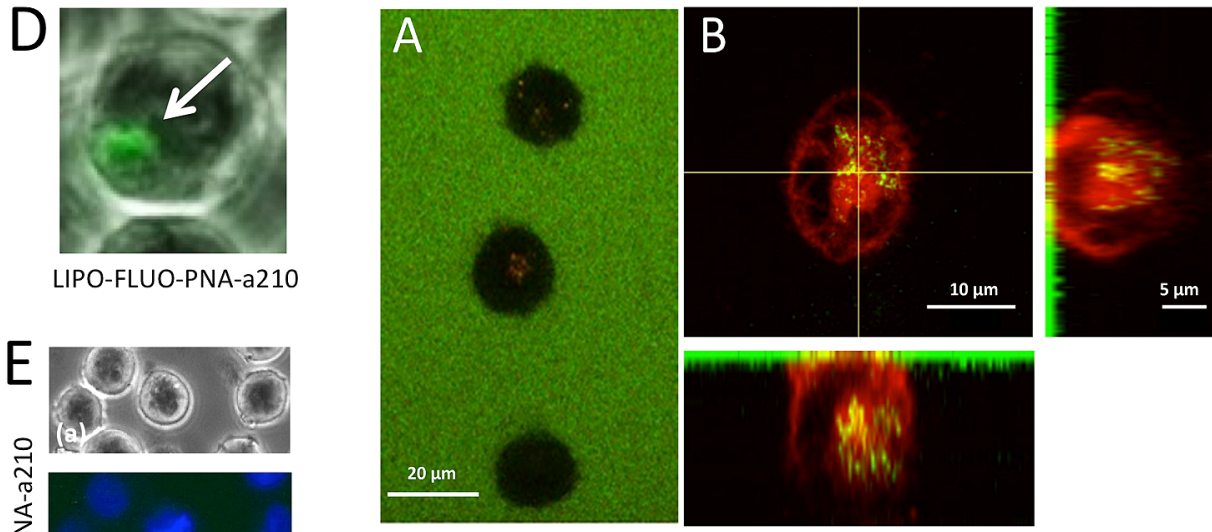

Figure 5. Uptake of PNA: confocal microscopy analyses. A. Representative confocal microscopic analysis of K562 cells cultured in the presence of FLUO-PNA-a210 $(24 \mathrm{~h}, 1 \mu \mathrm{M})$. B. Representative live images of $\mathrm{K} 562$ cells after $24 \mathrm{~h}$ incubation with $1 \mu \mathrm{M}$ Rho-LIPOFLUO-PNA-a210. Fluorescence profile was run across the $x-y$ axes of the cell body and orthogonal view of merged image was obtained with confocal Z-stack.

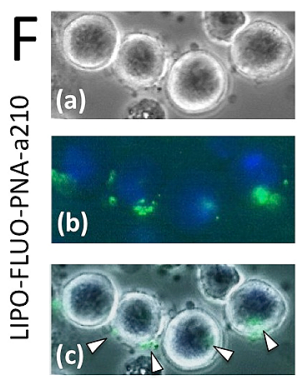

Figure 4. Uptake of PNAs. A,B. Representative FACS analysis of K562 cells cultured for $4 \mathrm{~h}$ in the presence of the indicated concentrations of FLUO-PNA-a210 (A), LIPO-FLUO-PNA-a210 (A), and FLUO-PNAa210-R8 (B). C. Kinetics of uptake of $2 \mu \mathrm{M}$ FLUO-PNA-a210 (open triangles), $4 \mu \mathrm{M}$ FLUO-PNA-a210 (black triangles), $2 \mu \mathrm{M}$ LIPOFLUO-PNA-a210 (open circles), and $4 \mu \mathrm{M}$ LIPO-FLUO-PNA-a210 (black circles). D. Representative microscopic analysis showing intracellular localization of LIPO-FLUO-PNA-a210 (arrowed) in K562 cells. E,F. Microscopic analysis of (a) K562 cells, phase-contrast analysis; (b) K 562 cells stained with DAPI and treated for $24 \mathrm{~h}$ with 1 $\mu \mathrm{M}$ FLUO-PNA-a210 (E) and LIPO-FLUO-PNA-a210 (F); (c) merge.

suggest that the liposomes facilitate cellular binding and uptake of FLUO-PNA-a210 molecules.

Effects of LIPO-PNA-a210 on miR-210 in K562 Cells. In order to determine whether the exposure to LIPO-PNA-a210 leads to alteration of miR-210 in K562 cells, RNA isolated from treated cells was used for RT-qPCR reactions amplifying miR210. We first determined the effects of the different treatments (48 h incubation) on cell growth (Figure S1).

Interestingly, the LIPO and the LIPO-PNA-a210 preparations caused only minor effects on the rate of cell growth, demonstrating lack of cytotoxic activity (Figure S1). When K562 cells were cultured in the presence of LIPO-PNA-a210 a very reproducible effect was obtained, as shown in Figure 6. The results demonstrate that the miR-210 specific hybridization signal was reduced when RNA was isolated from K562 cells cultured for $48 \mathrm{~h}$ in the presence of LIPO-PNA-a210 (Figure $6 \mathrm{~A})$.

On the contrary, the PNA-a210, lacking the R8 peptide, was not effective, in agreement with results published elsewhere. ${ }^{26}$ Notably, no effects were found using the liposomes carrying a scrambled PNA (LIPO-PNA-a210-scr) (Figure 6A). As positive
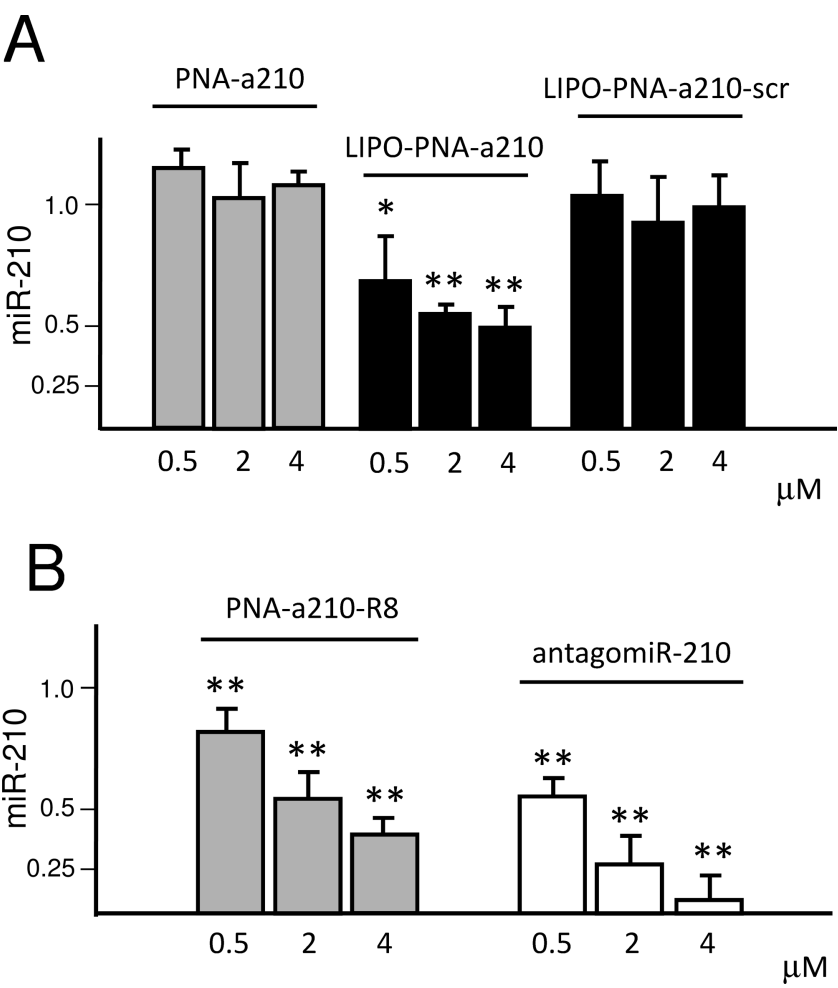

Figure 6. Real-time detection of miR-210 in K562 cells treated for 48 $\mathrm{h}$ with PNA-a210 (A), LIPO-PNA-a210 (A), LIPO-PNA-a210-scr (A), PNA-a210-R8 (B), and antagomiR-210 reference control (B), used at the indicated concentrations. RNA was extracted and RT-qPCR identifying miR-210 was performed and compared to control untreated K562 cells. Results represent the average \pm SD of three independent experiments $(*, p<0.05$, significant; $* *, p<0.01$, highly significant).

controls we employed the PNA-a210-R8 validated elsewhere and a commercially available antagomiR-210. ${ }^{26,27}$ The results obtained are shown in Figure 6B, and support the concept that the LIPO-PNA-a210 formulation approaches the activity of these already validated antimiR-210 reagents. With regard to specificity, we verified this issue by analyzing the levels of an 
additional microRNA, miR-221, in K562 cells treated with LIPO-PNA-a210. The data conclusively demonstrated that the effects of LIPO-PNA-a210 were selective, as no major effects were obtained in treated cells on miR-221 (Figure S2).

\section{DISCUSSION}

Liposomes have been successfully applied for the in vivo delivery of drugs and contrast agents; ${ }^{28-33}$ several liposomal formulations have been approved by FDA and are employed in the clinic for drug delivery in the cure of various diseases ranging from cancer (Doxil) to fungal infections (Ambisome) to age-related macular degeneration (Visudyne). ${ }^{34}$

The composition of the liposome, which determines its structural properties (size, external charge, polydispersity index, transition temperature, drug loading, and release), its stability in biological fluids, and also the ability to circulate for long times, is chosen based on the molecule that has to be encapsulated. The most used phospholipids for preparation of liposomal drugs including peptides are HSPC (L- $\alpha$-phosphatidylcholine, hydrogenated soy), DOPC (1,2-dioleoyl-sn-glycero-3-phosphocholine), DSPC (1,2-distearoyl-sn-glycero-3-phosphocholine), PC, DSPE (1,2-distearoyl-sn-glycero-3-phosphoethanolamine); all of them contain two saturated or unsaturated alkyl chains composed of 18 carbon atoms. In most preparations, cholesterol is added in order to favor membrane stabilization or a fluidizing effect on lipid bilayers. ${ }^{35}$ The introduction of flexible PEG chains exposed on the liposome surface has been reported to hide the liposome surface and prevent liposome interaction with MPS macrophages, thus providing stealth liposomes characterized by an increased circulation time. ${ }^{20}$ Instead, liposome/DNA complexes (Lipoplexes) are usually prepared using cationic lipids such as DOPE, DOTMA, and DOTAP. The positive surface charge favors the electrostatic interaction of the nucleic acid with the liposome.

PNA oligomers, presenting a peptide backbone and nucleotidic bases, can be considered hybrid molecules with intermediate features between a nucleic acid and a peptide; due to the lack of charges on the backbone, PNAs do not efficiently interact with cationic liposomes. For this reason we have investigated PNA encapsulation in a liposomal composition, reported to efficiently deliver peptides into cells, ${ }^{29}$ based on the zwitterionic egg PC, cholesterol, and DSPE-PEG2000, according to different experimental procedures, and the ability of the loaded vehicle to carry PNA oligomers inside cells. The PNA sequences were designed to be complementary to miR210 , a noncoding RNA whose expression can be induced by mithramycin, which is associated with erythroid differentiation of leukemic K562 cells, regulation of raptor and $\gamma$-globin genes. $^{36}$

We explored three different encapsulation techniques: thin film method/sonication and freeze/thawing method, which are usually employed for the entrapment of peptide derivatives in the liposomal inner compartment, and SNALPs method, which is preferred for the DNA or siRNA encapsulation. ${ }^{37}$ The freeze/thawing method was found to yield lower PDI of the liposomes, as compared to the other methods, and was therefore employed to encapsulate PNA oligomers for in vitro studies. A further advantage of this method is that it avoids the presence of traces of ethanol required during the SNALPs preparation, which are hardly removed and may be toxic to cells.

Encapsulation efficiency (EE\%) and encapsulation ratio (ER $\%)$ for PNA-antimiR210 in liposomes using the freeze/thawing method are $0.175 \%$ and $55 \%$, respectively. The values obtained using the other two methods reported above are very similar, and all of them are in good agreement with data reported in the literature for the encapsulation of siRNAs. ${ }^{38}$ On the contrary, a noticeable difference can be underlined for FLUO-PNAantimiR210 with respect to PNA-antimir210 with a decrease of EE\% from $1.70-1.85$ to $0.70-0.80$. This decrease in loading can be ascribed to the lower solubility of the fluorescently labeled derivative as compared to the unlabeled peptide nucleic acid. The encapsulation efficiency can be further improved ( $\sim 30 \%)$ by PEG post-insertion. This result is probably due to the reduction of the membrane permeability (pore formation) in the lipid bilayer as a consequence of introduction of the highly flexible PEG headgroup. Moreover, the PEG insertion favors a better packing of the water-hydrocarbon interface, which also could reduce the membrane permeability. Once prepared, the liposomes containing the PNAs were lyophilized and stored until usage. Furthermore, LIPO PNA-a210 are stable in serum over time, as shown in Figure 3. FACS measurements were carried out on fluorescent derivatives LIPO-FLUO-PNA-a210, FLUO-PNA-a210, and FLUO-PNAa210-R8. The FLUO-PNA-a210-R8 was used as positive control since this PNA is internalized by target cells with very high efficiency, as reported elsewhere. ${ }^{26}$ LIPO-FLUOPNA-a210 is taken up by cells more efficiently than FLUOPNA-a210, which does not enter into cells, and also than FLUO-PNA-a210-R8. This latest result is of particular relevance as it suggests that conjugation of PNA to carrier peptides is no longer needed to achieve uptake, as far as the correct liposomal formulation is employed. Moreover, the uptake of LIPO-FLUO-PNA-a210 occurs rapidly, only after $4 \mathrm{~h}$ incubation in $\mathrm{K} 562$ cells. As liposomal formulations, in particular, those possessing anionic and cationic lipids, might be, to some extent, potentially toxic, we investigated the growth of K562 cells upon incubation with PNA-a210, PNA-a210-R8, antagomiR-210, empty liposomes, LIPO-PNA-a210, and LIPOPNA-a210scr. The data obtained indicate that the LIPO, LIPOPNA-a210, and LIPO-PNA-a210-scr formulation at all the concentrations studied did not induce major alterations in cell growth indicating a lack of cytotoxicity (Figure S1). Finally, we investigated the ability of the formulation to down-regulate the target miR-210, to verify whether the internalization does not alter biological properties of the delivered PNA. LIPO-PNAa210, unlike PNA-a210, causes a reduction in the expression of miR-210 at $0.5 \mu \mathrm{M}$ PNA concentration. It is worth highlighting that no toxicity is observed at this concentration of liposomal formulation. This result further demonstrates that biological effects can be achieved at very low concentrations of PNAs, as far as the delivery is efficient. In addition, the extent of the effects of the liposome-delivered PNA resemble that of commercially available antagomiR-210, despite the fact that a comparison is not possible, due to the need for transfection reagents to allow antagomiR-210 internalization by target cells. As a final comment, we would like to emphasize that liposomedelivered PNA-a210 maintains its specificity on miR-210 downregulation, since the expression of the unrelated miR-221 is not affected by LIPO-PNA-a210 (Figure S2). Further efforts are needed to compare liposome-delivered PNAs with R8-delivered PNAs and commercially available antagomiRs, including the use of different cell lines, the design of anti-miR molecules with different length, PNA sequences targeting different miRNAs, and extensive studies on miRNA-regulated mRNAs. 


\section{CONCLUSIONS}

We have reported the first example of a liposomal PNA formulation, in which a PNA oligomer is efficiently loaded in a pegylated liposome composition based on the zwitterionic PC phospholipid, cholesterol, and DSPE-PEG2000. The PNA loaded vehicle is quickly and efficiently delivered inside K562 cells, keeping unmodified the biological properties of the delivered PNA. The first important implication of this result is that a low concentration of PNA is needed to trigger a biological effect. These results will open the way to the use of these oligonucleotide analogues, without further modifications, in in vitro and in vivo applications.

\section{MATERIALS AND METHODS}

Protected N-Fmoc-amino acid derivatives, acetic anhydride, and coupling reagents have been purchased at Novabiochem. Fmoc-PNA-cytosine(Bhoc)-OH, Fmoc-PNA-thymine-OH, Fmoc-PNA-guanine(Bhoc)-OH, and Fmoc-PNA-adenine(Bhoc)-OH were obtained from Link Technologies. Acetonitrile (ACN) for LC-MS, N,N-dimethylformamide (DMF) for solid phase synthesis, $N, N$-diisopropylethylamine (DIPEA), and dichloromethane (DCM) were from Romil Pure Chemistry, and piperidine from Biosolve. Fmoc-PAL-PEG-PS ( $0.16 \mathrm{mmol}$ ) g) resin was from Applied Biosystems. All other chemicals were supplied by Sigma-Aldrich and were used without further purification.

Egg phosphatidylcholine (Egg PC; purity, 98\%) cholesterol (Chol; purity > 99\%) 1,2-distearoyl-sn-glycero-3-phosphoethanolamine- $N$-[ carbonyl-methoxy(polyethylene glycol)2000] (ammonium salt) (DSPE-PEG2000) and Rhod-PE (1,2dioleoyl-sn-glycero-3-phosphoethanolamine- $N$-(Lissamine Rhodamine B sulfonyl) ammonium salt) were purchased at Avanti Polar Lipids (Alabaster, AL).

LC-MS analyses were performed on a LC-MS Agilent Technologies 6230 ESI-TOF on a Phenomenex Jupiter $3 \mu$ $\mathrm{C} 18(150 \times 2.0 \mathrm{~mm})$ column with a flow rate of $0.2 \mathrm{~mL} \cdot \mathrm{min}^{-1}$.

PNA Synthesis. The PNA oligomers were synthesized on a $2 \mu \mathrm{mol}$ scale using the standard protocol for the solid phase synthesis of PNA. ${ }^{39,40}$ PNA oligomers were purified by RPHPLC using a gradient of acetonitrile (0.1\% TFA) in water (0.1\% TFA) from $5 \%$ to $50 \%$ in $30 \mathrm{~min}$ and characterized by ESI-TOF, employing a gradient of acetonitrile (0.05\% TFA) in water $(0.05 \%$ TFA) from $5 \%$ to $50 \%$ applied over $30 \mathrm{~min}$.

PNA antimir210 (PNA-a210) sequence: H-ccgctgtcacacgcacag- $\mathrm{NH}_{2}$. Mass calculated (Da): 4825.67; $[\mathrm{M}+3 \mathrm{H}]^{3+}: 1609.55$ $[\mathrm{M}+4 \mathrm{H}]^{4+}: 1207.41$; found: $[\mathrm{M}+3 \mathrm{H}]^{3+}: 1609.32 ;[\mathrm{M}+4 \mathrm{H}]^{4+}$ : 1207.24; $[\mathrm{M}+5 \mathrm{H}]^{5+}: 965.99$.

PNA antimiR210 scramble (PNA-a210 scr) sequence: Hagcgacgcgatcctcacc- $\mathrm{NH}_{2}$ Mass calculated (Da): 4825.67; [M $+3 \mathrm{H}]^{3+}: 1609.55[\mathrm{M}+4 \mathrm{H}]^{4+}: 1207.41 ;[\mathrm{M}+5 \mathrm{H}]^{5+}: 966.16$; found: 4824.98; $[\mathrm{M}+3 \mathrm{H}]^{3+}: 1609.32 ;[\mathrm{M}+4 \mathrm{H}]^{4+}: 1207.24 ;[\mathrm{M}$ $+5 \mathrm{H}]^{5+}: 965.99$.

PNA antimiR210-R 8 (PNA-a210-R8) Conjugate Synthesis. To obtain the PNA-peptide conjugate, we first synthesized the peptide on solid phase following standard Fmoc chemistry protocol on a Fmoc-PAL-PEG-PS resin (0.16 $\mathrm{mmol} / \mathrm{g}$ ). The PNA was grown on the peptide anchored to the solid phase, following procedures reported above. The conjugate was cleaved from the resin and deprotected by treatment with a solution of $78 \%$ TFA, $m$-cresol $20 \%$, and $2 \%$ triisopropylsilane for $3 \mathrm{~h}$ at room temperature. The conjugate was purified by RP-HPLC using a gradient of acetonitrile $(0.1 \%$
TFA) in water (0.1\% TFA) from $5 \%$ to $50 \%$ in $30 \mathrm{~min}$ and characterized by ESI-TOF employing a gradient of acetonitrile (0.05\% TFA) in water (0.05\% TFA) from 5\% to $50 \%$ applied over $30 \mathrm{~min}$.

PNA antimir210- $R_{8}$ (PNA-a210-R8) sequence: H-ccgctgtcacacgcacag-RRRRRRRR-NH ${ }_{2}$. Mass calculated (Da): 6074.68; $[\mathrm{M}+4 \mathrm{H}]^{4+}: 1519.67 ;[\mathrm{M}+5 \mathrm{H}]^{5+}: 1215.94 ;[\mathrm{M}+6 \mathrm{H}]^{6+}: 1013.44$; found: $[\mathrm{M}+4 \mathrm{H}]^{4+}: 1519.25[\mathrm{M}+5 \mathrm{H}]^{5+}: 1215.60[\mathrm{M}+6 \mathrm{H}]^{6+}$ : 1013.16.

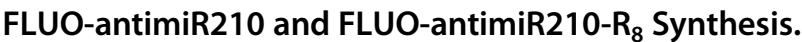
Derivatization of the PNA and PNA-peptide conjugate with fluorescein was carried out on the solid phase. To the resinbound oligomers, the fluorescein-5(6)-carboxamidocaproic acid (FLUO-Ahx-OH) was coupled following standard peptide synthesis protocols for $1 \mathrm{~h}$, two times. Cleavage and deprotection of the conjugates were performed as described earlier. Purification and characterization were performed following the protocols described for peptide-PNA conjugates.

FLUO-PNA antimir210 (FLUO-PNA-a210) sequence: FLUO-Ahx-ccgctgtcacacgcacag- $\mathrm{NH}_{2}$. Mass calculated (Da): 5327.46; $[\mathrm{M}+3 \mathrm{H}]^{3+}: 1776.82 ;[\mathrm{M}+4 \mathrm{H}]^{4+}: 1332.86 ; \quad[\mathrm{M}$ $+5 \mathrm{H}]^{5+}: 1066.49$; found: $[\mathrm{M}+3 \mathrm{H}]^{3+}:$ 1766.36; $[\mathrm{M}+4 \mathrm{H}]^{4+}$ : 1325.02; $[\mathrm{M}+5 \mathrm{H}]^{5+}: 1060.42$.

FLUO-PNA antimir210-R (FLUO-PNA-a210-R8) sequence: FLUO-Ahx-ccgctgtcacacgcacag-RRRRRRRR- $\mathrm{NH}_{2}$. Mass calculated (Da): 6576.46; $[\mathrm{M}+4 \mathrm{H}]^{4+}: 1645.11 ;[\mathrm{M}$ $+5 \mathrm{H}]^{5+}:$ 1316.29; $[\mathrm{M}+6 \mathrm{H}]^{6+}: 1097.08 ;$ found: $[\mathrm{M}+4 \mathrm{H}]^{4+}$ : $1644.99[\mathrm{M}+5 \mathrm{H}]^{5+}: 1316.19[\mathrm{M}+6 \mathrm{H}]^{6+}: 1096.99$.

Liposome Preparation. Three different protocols were tested to obtain PNA loaded liposomes. The same liposomal formulation, composed of egg PC/Chol/DSPE-PEG2000 (47/ $47 / 6$, molar \% ratio) was employed for all the protocols. Stock solutions of PNA-antimiR210 and of its fluorescent derivative FLUO-PNA-antimiR210 were prepared in $10 \mathrm{mM}$ phosphate buffer at $\mathrm{pH} 7.4$, containing the 0.9 wt $\% \mathrm{NaCl}$ and $100 \mathrm{mM}$ glycerol. Concentrations of all PNA solutions were determined by UV-vis measurements, carried out on Thermo Fisher Scientific Inc. (Wilmington, Delaware USA) Nanodrop 2000c spectrophotometer equipped with a $1.0 \mathrm{~cm}$ quartz cuvette (Hellma) using a molar absorptivity $(\varepsilon)$ of $172400 \mathrm{M}^{-1} \mathrm{~cm}^{-1}$ at $\lambda=260 \mathrm{~nm}$. Fluorescent liposomes were prepared by introducing in the formulation $0.1 \% \mathrm{~mol} / \mathrm{mol}$ of the fluorescent probe Rhod-PE.

(a). Thin Film Method/Sonication Method. The required amounts of phospholipids (10.8 mg of PC and $5.0 \mathrm{mg}$ of DSPE-PEG2000) and cholesterol $(5.4 \mathrm{mg})$ were dissolved in 3 $\mathrm{mL}$ of a methanol/chloroform (50/50) mixture; subsequently, the organic solvents were removed under a stream of nitrogen gas to obtain a homogeneous film on the wall of the vial. Any trace solvent was then removed keeping the vial under vacuum for $15 \mathrm{~min}$. Then, the dry lipid film was hydrated with $3 \mathrm{~mL}$ of a solution containing $500 \mu \mathrm{g}$ of PNA-antimiR210 or FLUOPNA-antimiR210 and sonicated in an ultrasound bath for 30 min. The liposomal suspension was extruded 10 times at room temperature, using a thermobarrel extruder system (Northern Lipids Inc., Vancouver, BC, Canada) under nitrogen through a polycarbonate membrane (Nucleopore Track Membrane 25 mm, Whatman, Brentford, UK) with $0.1 \mu \mathrm{m}$ pore size.

(b). Freeze/Thawing Method. Dry lipid film was prepared as reported above, dissolving phospholipids in a methanol/ chloroform (50/50) mixture. Then, the lipid film was hydrated with PNA solutions (500 $\mu \mathrm{g}$ of either PNA-a210 and FLUOPNA-a210 in $3 \mathrm{~mL}$ of buffer), and the resulting liposomes were 
frozen/thawed dipping the glass tube for $1 \mathrm{~min}$ into a dry ice bath for rapid cooling. The frozen sample was transferred into a bath at $65{ }^{\circ} \mathrm{C}$, allowing it to thaw out for $4 \mathrm{~min}$, and vortexed for another minute. Ten cycles were performed to promote the entry of PNAs into the liposomes.

(c). SNALP Method. PNA loaded liposomes were prepared using the spontaneous vesicle formation by ethanol dilution method. Lipids $(30.0 \mu \mathrm{mol}$ in $1 \mathrm{~mL}$ of ethanol) and PNA (either PNA-a210 and FLUO-PNA-a210, $500 \mu \mathrm{g}$ in $3 \mathrm{~mL}$ of the buffer) solutions were separately prepared and warmed at 50 ${ }^{\circ} \mathrm{C}$ for $5 \mathrm{~min}$. After warming, the lipid solution was rapidly added to the PNA solution and then the suspension was extruded 10 times at $50{ }^{\circ} \mathrm{C}$ with $0.1 \mu \mathrm{m}$ pore size.

For all methods, the unencapsulated PNA-antimiR210 or FLUO-PNA-antimiR210 were removed from the liposomal suspension using a Sephadex G50 gel filtration column, preequilibrated with $10 \mathrm{mM}$ phosphate buffer at $\mathrm{pH}$ 7.4, containing the 0.9 wt $\% \mathrm{NaCl}$ and $100 \mathrm{mM}$ glycerol. The amount of unloaded PNA eluted from the column was spectroscopically determined by UV-vis measurements at $\lambda$ $=260 \mathrm{~nm}$. The encapsulation efficiency $(\mathrm{EE} \%$, defined as the weight ratio of encapsulated PNA versus the amphiphilic molecules forming liposomes) and the encapsulation ratio (ER $\%$, defined as the weight percentage of PNA encapsulated in the liposomes on the total PNA previously added during preparation) were quantified by subtraction of the amount of removed PNA from the total amount of loaded PNA. Liposomes containing PNAs were lyophilized and stored at $-20{ }^{\circ} \mathrm{C}$. The integrity of the reconstituted liposomes was assessed by DLS measurements. The leakage of PNA from the liposome was verified loading the reconstituted liposomes on a gel-filtration column and measuring the absorbance of the eluates at $260 \mathrm{~nm}$.

DSPE-PEG2000 Post-Insertion. PC/Chol (47/47 molar \% ratio) liposomes were prepared and preloaded with PNA-a210 or FLUO-PNA-a210 by the freeze/thawing method. Liposomes were incubated with an aqueous micellar solution of DSPEPEG2000 $(6 \% \mathrm{~mol})$ for $2 \mathrm{~h}$ at room temperature under stirring. $^{41}$

DLS Measurements. Hydrodynamic radii (RH), polydispersity indexes (P.I.) and $\mathrm{Z}$ potentials $(\zeta)$ of filled and empty liposomes were measured by dynamic light scattering technique (DLS). DLS measurements were carried out using a Zetasizer Nano ZS (Malvern Instruments, Westborough, MA) that employs a $173^{\circ}$ backscatter detector. Other instrumental settings are measurement position $(\mathrm{mm}), 4.65$; attenuator, 8; temperature, $25{ }^{\circ} \mathrm{C}$; cell, disposable sizing cuvette. DLS measurements were carried out on samples previously prepared, after centrifugation at room temperature at 13000 rpm for $5 \mathrm{~min}$ at $2.0 \times 10^{-4} \mathrm{M}$ final lipid concentration. For each formulation, both hydrodynamic radii, polydispersity indexes, and $\mathrm{Z}$ potentials were calculated as the mean of at least three measurements on three different batches.

Serum Stability. PNA-antimiR210 loaded liposomes (LIPO-PNA-a210) were suspended in $10 \mathrm{mM}$ phosphate buffer at $\mathrm{pH} 7.4$ containing $0.9 \% \mathrm{NaCl}$ at $10 \mathrm{mM}$ lipid concentration and then diluted at $1 \mathrm{mM}$ with fetal bovine serum (FBS; Biowest, Nuaillé, France). The colloidal suspension was stirred at $37{ }^{\circ} \mathrm{C}$ for $72 \mathrm{~h}$. Samples withdrawn at different time points $(2,4,8,12,28,36,48$, and $72 \mathrm{~h})$ were diluted with PBS (1:50 v/v) and analyzed by dynamic light scattering as described earlier.
PNA Uptake by K562 Cells. To determine the PNA uptake, flow cytometry analyses (BD FACScan) were performed. In order to observe uptake kinetics, K562 cells were seeded at initial concentrations of $3 \times 10^{5}$ per well in 24 well plates for 4,24 , and $48 \mathrm{~h}$. Cells were grown at $37^{\circ} \mathrm{C}$ in $5 \%$ $\mathrm{CO}_{2}$ in fresh culture medium RPMI 1640 (Lonza BioWhittaker, Basel, Switzerland) supplemented with 10\% FBS and incubated with PNA-a210, PNA-a210-R8, or LIPO-PNAa210 at final concentrations of $2 \mu \mathrm{M}$ and $4 \mu \mathrm{M}$. After the treatment, cells were centrifuged at $1800 \mathrm{rpm}$ for $5 \mathrm{~min}$, washed in PBS (Lonza BioWhittaker, Basel, Switzerland) with 1\% FBS, and suspended in $200 \mu \mathrm{L}$ PBS with 1\% FBS. For each sample, 30000 events were analyzed and fluorescence emission at 515545 nm (FL1) was detected, using BD Cell Quest Pro. The intracellular distribution of FLUO-LIPO-PNA-a210 was first studied by microscopy analysis of living cells using the BioStation instrument, a compact cell incubation and monitoring system (BioStation IM, Nikon Instruments Europe B.V., Italy), previously demonstrated to be a suitable system to detect intracellular distribution of fluorescein-labeled PNAs. ${ }^{25,42}$ Then, the cells were fixed, counterstained with $4 \alpha, 6$-diamidino-2-phenylindole (DAPI) ${ }^{25}$ for nuclear labeling, and further analyzed. Live images of cells treated with FLUOPNA-a210 and Rho-LIPO-FLUO-PNA-a210 were also obtained using a Nikon Swept Field Confocal equipped with CFI Plan Apo VC60XH objective (numerical aperture, 1.4) (Nikon Instruments, Melville, NY, USA) and an Andor DU885 electron multiplying charge-coupled device (EM-CCD) camera (Andor Technology Ltd., Belfast, Northern Ireland). Acquired images were then analyzed by using open source software Fiji. $^{43,44}$

Transfection Protocols for Standard RNA antagomiR. The transfection of the anti-microRNAs was carried out following the protocol reported by Ambion (Applied Biosystems, Foster City, CA, USA) and described elsewhere. ${ }^{27}$ $30000 \mathrm{~K} 562 \mathrm{cell} / \mathrm{mL}$ were seeded in 24-well plates. Treatments with increasing concentrations of antagomiR-210 (AM105116 Applied Biosystems, Foster City, CA, USA) were performed using the siPort NeoFX transfection reagent.

Quantitative Analyses of miRNAs. Reverse transcriptase (RT) reactions were performed using the TaqMan MicroRNA Reverse Transcription Kit (Applied Biosystems, Foster City, CA, USA); real-time PCR was performed according to the manufacturer's protocols. $500 \mathrm{ng}$ of total RNA per sample were used for the assays. All RT reactions, including no-template controls and RT-minus controls, were performed in duplicate using the CFX96 Touch Real-Time PCR Detection System (Bio-Rad Laboratories, Milan, Italy). The relative expression was calculated using the comparative cycle threshold method and as reference U6 snRNA was used to normalize all RNA samples, since it remains constant in the assayed samples by miR-profiling and quantitative RT-PCR analysis, as previously reported. $^{45}$

Statistical Analysis. All the data were normally distributed and presented as mean \pm SD. Statistical differences between groups were compared using one-way ANOVA (analyses of variance between groups) software. Statistical differences were considered significant when $p<0.05(*)$, highly significant when $p<0.01(* *)$. 


\section{ASSOCIATED CONTENT}

\section{S Supporting Information}

Additional figures and tables. The Supporting Information is available free of charge on the ACS Publications website at DOI: 10.1021/acs.bioconjchem.5b00156.

\section{AUTHOR INFORMATION}

\section{Corresponding Author}

*E-mail: alessandra.romanelli@unina.it.

\section{Author Contributions}

${ }^{\#}$ Concetta Avitabile and Antonella Accardo equally contributed to the work.

\section{Notes}

The authors declare no competing financial interest.

\section{ACKNOWLEDGMENTS}

The authors thank Mr. Maurizio Amendola, Dr. Lucrezia Cassano, Dr. Caterina Chiarella, and Mr. Giovanni Nasti for technical/administrative assistance. This work was supported by the Fondazione CON IL SUD (2011-PDR-20) and by the Italian Ministry for Research (M.I.U.R.) FIRB "RENAME" RBAP114AMK. R.G. is supported by AIRC (IG 13575: peptide nucleic acids targeting oncomiR and tumor-suppressor miRNAs: cancer diagnosis and therapy) and by UE FP7 THALAMOSS (Thalassemia Modular Stratification System for Personalized Therapy of B-Thalassemia; n.306201-FP7HEALTH-2012- INNOVATION-1).

\section{REFERENCES}

(1) Avitabile, C., Cimmino, A., and Romanelli, A. (2014) Oligonucleotide Analogues as Modulators of the Expression and Function of Noncoding RNAs (ncRNAs): Emerging Therapeutics Applications. J. Med. Chem. 57, 10220-40.

(2) Nielsen, P. E., Egholm, M., Berg, R. H., and Buchardt, O. (1991) Sequence-selective recognition of DNA by strand displacement with a thymine-substituted polyamide. Science 254, 1497-500.

(3) Egholm, M., Buchardt, O., Christensen, L., Behrens, C., Freier, S. M., Driver, D. A., Berg, R. H., Kim, S. K., Norden, B., and Nielsen, P. E. (1993) PNA hybridizes to complementary oligonucleotides obeying the Watson-Crick hydrogen-bonding rules. Nature 365, 566-8.

(4) Nielsen, P. E. (2010) Gene targeting and expression modulation by peptide nucleic acids (PNA). Curr. Pharm. Des. 16, 3118-23.

(5) Borgatti, M., Lampronti, I., Romanelli, A., Pedone, C., Saviano, M., Bianchi, N., Mischiati, C., and Gambari, R. (2003) Transcription factor decoy molecules based on a peptide nucleic acid (PNA)-DNA chimera mimicking Sp1 binding sites. J. Biol. Chem. 278, 7500-9.

(6) Gambari, R., Fabbri, E., Borgatti, M., Lampronti, I., Finotti, A., Brognara, E., Bianchi, N., Manicardi, A., Marchelli, R., and Corradini, R. (2011) Targeting microRNAs involved in human diseases: a novel approach for modification of gene expression and drug development. Biochem. Pharmacol. 82, 1416-29.

(7) Gambari, R. (2014) Peptide nucleic acids: a review on recent patents and technology transfer. Expert Opin. Ther. Pat. 24, 267-94.

(8) Piva, R., Spandidos, D. A., and Gambari, R. (2013) From microRNA functions to microRNA therapeutics: novel targets and novel drugs in breast cancer research and treatment (Review). Int. J. Oncol. 43, 985-94.

(9) Pensato, S., Saviano, M., and Romanelli, A. (2007) New peptide nucleic acid analogues: synthesis and applications. Expert Opin. Biol. Ther. 7, 1219-32.

(10) Roviello, G. N., Musumeci, D., Bucci, E. M., and Pedone, C. (2012) Synthesis of a diaminopropanoic acid-based nucleoamino acid and assembly of cationic nucleopeptides for biomedical applications. Amino Acids 43, 2537-43.
(11) Roviello, G. N., Musumeci, D., D’Alessandro, C., and Pedone, C. (2013) Synthesis of a thymine-functionalized nucleoamino acid for the solid phase assembly of cationic nucleopeptides. Amino Acids 45, 779-84.

(12) Dragulescu-Andrasi, A., Zhou, P., He, G., and Ly, D. H. (2005) Cell-permeable GPNA with appropriate backbone stereochemistry and spacing binds sequence-specifically to RNA. Chem. Commun. (Cambridge, U. K.), 244-6.

(13) Dragulescu-Andrasi, A., Rapireddy, S., He, G., Bhattacharya, B., Hyldig-Nielsen, J. J., Zon, G., and Ly, D. H. (2006) Cell-permeable peptide nucleic acid designed to bind to the 5 -untranslated region of E-cadherin transcript induces potent and sequence-specific antisense effects. J. Am. Chem. Soc. 128, 16104-12.

(14) Shiraishi, T., Hamzavi, R., and Nielsen, P. E. (2008) Subnanomolar antisense activity of phosphonate-peptide nucleic acid (PNA) conjugates delivered by cationic lipids to HeLa cells. Nucleic Acids Res. 36, 4424-32.

(15) Capparelli, R., De Chiara, F., Nocerino, N., Montella, R. C., Iannaccone, M., Fulgione, A., Romanelli, A., Avitabile, C., Blaiotta, G., and Capuano, F. (2012) New perspectives for natural antimicrobial peptides: application as antinflammatory drugs in a murine model. BMC Immunol. 13, 61.

(16) de Koning, M. C., van der Marel, G. A., and Overhand, M. (2003) Synthetic developments towards PNA-peptide conjugates. Curr. Opin. Chem. Biol. 7, 734-40.

(17) Cheng, C. J., Bahal, R., Babar, I. A., Pincus, Z., Barrera, F., Liu, C., Svoronos, A., Braddock, D. T., Glazer, P. M., Engelman, et al. (2014) MicroRNA silencing for cancer therapy targeted to the tumour microenvironment. Nature 518, 107-10.

(18) Ma, X., Devi, G., Qu, Q., Toh, D. F., Chen, G., and Zhao, Y. (2014) Intracellular delivery of antisense peptide nucleic acid by fluorescent mesoporous silica nanoparticles. Bioconjugate Chem. 25, 1412-20.

(19) McNeer, N. A., Schleifman, E. B., Cuthbert, A., Brehm, M., Jackson, A., Cheng, C., Anandalingam, K., Kumar, P., Shultz, L. D., Greiner, D. L., et al. (2013) Systemic delivery of triplex-forming PNA and donor DNA by nanoparticles mediates site-specific genome editing of human hematopoietic cells in vivo. Gene Ther. 20, 658-69.

(20) Immordino, M. L., Dosio, F., and Cattel, L. (2006) Stealth liposomes: review of the basic science, rationale, and clinical applications, existing and potential. Int. J. Nanomedicine 1, 297-315.

(21) Janowski, B. A., Kaihatsu, K., Huffman, K. E., Schwartz, J. C., Ram, R., Hardy, D., Mendelson, C. R., and Corey, D. R. (2005) Inhibiting transcription of chromosomal DNA with antigene peptide nucleic acids. Nat. Chem. Biol. 1, 210-5.

(22) Borgatti, M., Breda, L., Cortesi, R., Nastruzzi, C., Romanelli, A., Saviano, M., Bianchi, N., Mischiati, C., Pedone, C., and Gambari, R. (2002) Cationic liposomes as delivery systems for double-stranded PNA-DNA chimeras exhibiting decoy activity against NF-kappaB transcription factors. Biochem. Pharmacol. 64, 609-16.

(23) Nastruzzi, C., Cortesi, R., Esposito, E., Gambari, R., Borgatti, M., Bianchi, N., Feriotto, G., and Mischiati, C. (2000) Liposomes as carriers for DNA-PNA hybrids. J. Controlled Release 68, 237-49.

(24) Manicardi, A., Fabbri, E., Tedeschi, T., Sforza, S., Bianchi, N., Brognara, E., Gambari, R., Marchelli, R., and Corradini, R. (2012) Cellular uptakes, biostabilities and anti-miR-210 activities of chiral arginine-PNAs in leukaemic K562 cells. ChemBioChem 13, 1327-37.

(25) Brognara, E., Fabbri, E., Bazzoli, E., Montagner, G., Ghimenton, C., Eccher, A., Cantu, C., Manicardi, A., Bianchi, N., Finotti, A., et al. (2014) Uptake by human glioma cell lines and biological effects of a peptide-nucleic acids targeting miR-221. J. Neuro-Oncol. 118, 19-28.

(26) Fabbri, E., Manicardi, A., Tedeschi, T., Sforza, S., Bianchi, N., Brognara, E., Finotti, A., Breveglieri, G., Borgatti, M., Corradini, R, et al. (2011) Modulation of the biological activity of microRNA-210 with peptide nucleic acids (PNAs). ChemMedChem 6, 2192-202.

(27) Bianchi, N., Finotti, A., Ferracin, M., Lampronti, I., Zuccato, C., Breveglieri, G., Brognara, E., Fabbri, E., Borgatti, M., Negrini, M., and Gambari, R. (2015) Increase of microRNA-210, decrease of raptor gene expression and alteration of mammalian target of rapamycin 
regulated proteins following mithramycin treatment of human erythroid cells. PLoS One 10, e0121567.

(28) Riche, E. L., Erickson, B. W., and Cho, M. J. (2004) Novel longcirculating liposomes containing peptide library-lipid conjugates: synthesis and in vivo behavior. J. Drug Target 12, 355-61.

(29) Ducat, E., Brion, M., Lecomte, F., Evrard, B., and Piel, G. (2010) The experimental design as practical approach to develop and optimize a formulation of peptide-loaded liposomes. AAPS PharmSciTech 11, 966-75.

(30) Lao, J., Madani, J., Puertolas, T., Alvarez, M., Hernandez, A., Pazo-Cid, R., Artal, A., and Anton Torres, A. (2013) Liposomal Doxorubicin in the treatment of breast cancer patients: a review. J. Drug Delivery 2013, 1.

(31) Martin, C., Low, W. L., Gupta, A., Amin, M. C. I. M., Radecka, I., Raj, P., Britland, S., and Kenward, K. (2014) Liposomal delivery of antimicrobial agents in advances in liposome research, Nova Science Publisher.

(32) Bakandritsos, A., Fatourou, A. G., and Fatouros, D. G. (2012) Magnetoliposomes and their potential in the intelligent drug-delivery field. Ther. Delivery 3, 1469-82.

(33) Accardo, A., Tesauro, D., Aloj, L., Pedone, C., and Morelli, G. (2009) Supramolecular aggregates containing lipophilic Gd(III) complexes as contrast agents in MRI. Coord. Chem. Rev. 253, 21932213.

(34) Zhang, Y., Chan, H. F., and Leong, K. W. (2013) Advanced materials and processing for drug delivery: the past and the future. Adv. Drug Delivery Rev. 65, 104-20.

(35) Coderch, L., Fonollosa, J., De Pera, M., Estelrich, J., De La Maza, A., and Parra, J. L. (2000) Influence of cholesterol on liposome fluidity by EPR. Relationship with percutaneous absorption. J. Controlled Release 68, 85-95.

(36) Bianchi, N., Zuccato, C., Lampronti, I., Borgatti, M., and Gambari, R. (2009) Expression of miR-210 during erythroid differentiation and induction of gamma-globin gene expression. $B M B$ Rep 42, 493-9.

(37) Morrissey, D. V., Lockridge, J. A., Shaw, L., Blanchard, K., Jensen, K., Breen, W., Hartsough, K., Machemer, L., Radka, S., Jadhav, V., et al. (2005) Potent and persistent in vivo anti-HBV activity of chemically modified siRNAs. Nat. Biotechnol. 23, 1002-7.

(38) Heyes, J., Palmer, L., Bremner, K., and MacLachlan, I. (2005) Cationic lipid saturation influences intracellular delivery of encapsulated nucleic acids. J. Controlled Release 107, 276-87.

(39) Avitabile, C., Fabbri, E., Bianchi, N., Gambari, R., and Romanelli, A. (2012) Inhibition of miRNA maturation by Peptide Nucleic Acids, in MiRNA maturation, Methods and Protocols (Arenz, C., Ed.) pp 157-164, Humana Press.

(40) Avitabile, C., Saviano, M., D’Andrea, L., Bianchi, N., Fabbri, E., Brognara, E., Gambari, R., and Romanelli, A. (2012) Targeting premiRNA by peptide nucleic acids: a new strategy to interfere in the miRNA maturation. Artif DNA PNA XNA 3, 88-96.

(41) Uster, P. S., Allen, T. M., Daniel, B. E., Mendez, C. J., Newman, M. S., and Zhu, G. Z. (1996) Insertion of poly(ethylene glycol) derivatized phospholipid into pre-formed liposomes results in prolonged in vivo circulation time. FEBS Lett. 386, 243-6.

(42) Brognara, E., Fabbri, E., Bianchi, N., Finotti, A., Corradini, R., and Gambari, R. (2014) Molecular methods for validation of the biological activity of peptide nucleic acids targeting microRNAs. Methods Mol. Biol. 1095, 165-76.

(43) Franceschini, A., Capece, M., Chiozzi, P., Falzoni, S., Sanz, J. M., Sarti, A. C., Bonora, M., Pinton, P., and Di Virgilio, F. (2015) The P2 $\times 7$ receptor directly interacts with the NLRP3 inflammasome scaffold protein. FASEB J. 29, 2450-61.

(44) Venco, P., Bonora, M., Giorgi, C., Papaleo, E., Iuso, A., Prokisch, H., Pinton, P., and Tiranti, V. (2015) Mutations of C19orf12, coding for a transmembrane glycine zipper containing mitochondrial protein, cause mis-localization of the protein, inability to respond to oxidative stress and increased mitochondrial Ca (2.). Front. Genet. 6, 185.

(45) Brognara, E., Fabbri, E., Aimi, F., Manicardi, A., Bianchi, N., Finotti, A., Breveglieri, G., Borgatti, M., Corradini, R., Marchelli, R., et al. (2012) Peptide nucleic acids targeting miR-221 modulate p27Kip1 expression in breast cancer MDA-MB-231 cells. Int. J. Oncol. 41, 2119-27. 